\title{
Éditorial
}

\section{Un nuage passe...}

Le volcan Eyjafjöll, qui évoque davantage un fauteuil ou un canapé IKEA que la plus grande crise du transport aérien, a redonné toute leur actualité à maintes discussions que nous avons eues et que nous avons encore dans le domaine de la radioprotection.

Le premier point concerne la différence entre le principe de précaution et le principe de prévention. Je pense qu'il faudrait se poser cette délicate question pour savoir où exactement se situe la problématique de la Radioprotection; prévention ou précaution? Si l'on reprend les propos tenus pendant cette crise aérienne, nous sommes sans doute plus près de la prévention que de la précaution, compte tenu de nos nombreuses connaissances sur les effets des faibles doses. Les volumineux rapports de l'UNSCEAR, si l'on s'en tient à ce seul organisme international, en témoignent. Ce point mérite réflexion, en particulier sur ce qui différencie ceux qui pensent « prévention » mais évoquent « précaution »...

Le second point intéressant dans cette crise du transport aérien, est la suprématie accordée pendant 4 à 5 jours à la modélisation aux dépens de la mesure. Les autorités ont affirmé qu'aucun avion ne pouvait décoller car le panache de cendres se déplaçait au-dessus de nos têtes, mais il a fallu attendre 4 à 5 jours pour que ces mêmes autorités décident d'envoyer des avions « renifleurs » pour faire des mesures... restées négatives. Là encore, c'est un point à méditer, bien que, en radioprotection, nous soyons beaucoup mieux armés, au niveau de la mesure...

Le troisième point concerne la granulométrie de ces poussières et, là aussi, on peut souligner que les lois de la physique des aérosols ne s'appliquent pas seulement aux produits radioactifs. Je pense que les cendres d'un nuage, même au nom imprononçable, obéissent aux mêmes lois. Une relecture d'un livre de base, celui de Renoux et Boulaud (Tec\&Doc, 1998) s'impose donc. Quant à l'inhalation de ces poussières, le modèle respiratoire de la CIPR (ICRP Publication 66, 1994) s'applique tout autant à ces particules volcaniques qu'à celles évoquées dans cette publication de référence.

Tirons-nous les leçons idoines des autres disciplines ou vivons-nous chacun dans notre pré carré ? C'est là, bien sûr, un autre point à méditer...

Enfin, que dire des préjugés. Beaucoup d'entre nous ont pensé ces dernières semaines à un autre nuage, en fait un panache, qui s'est promené au-dessus de l'Europe pendant plusieurs jours, il y a 24 ans. Pour preuve, des experts en aéronautique, pourtant avisés, ont ressorti les mêmes poncifs relatant l'arrêt du nuage de Tchernobyl à nos frontières ! Albert Einstein le disait fort justement, il y a un siècle déjà : «Il est plus facile de briser un préjugé qu'un atome »... 
Citons alors le prince du nuage :

«Tout ce que nous voyons cache quelque chose d'autre. »

René Magritte

Les voyages aériens étant devenus une préoccupation majeure, la description des scanners à rayons $\mathrm{X}$ que de nombreux pays veulent installer dans les aéroports s'impose. Ce numéro en rend compte.

Fini donc les yeux tournés vers les nuages ! Il est temps de regarder la feuille blanche et de rédiger un très bon article...

H. Métivier

Président du Comité de rédaction

\section{Hommage à Jean-Jacques Radecki...}

$\mathrm{Au}$ moment où nous allions mettre sous presse, nous avons appris le décès de notre collègue et ami JeanJacques Radecki, le 12 mai 2010 à l'âge de 55 ans. Membre du conseil d'administration de la SFRP, nous avons tous pu apprécier les grandes qualités professionnelles dans l'homme de convictions qu'il était et ses immenses qualités humaines. Dans les échanges de courriels que nous avons eu dès l'annonce de cette

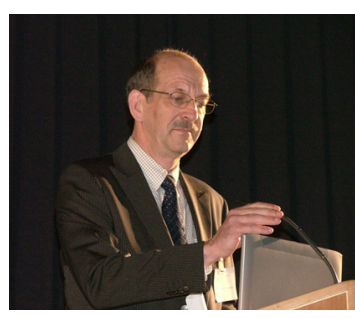
triste nouvelle, nous déplorons tous la perte d'un grand spécialiste de la radioprotection, un excellent ami et un membre très actif de notre société.

Le Comité de rédaction et la Société française de radioprotection transmettent à son épouse et à ses enfants leurs plus sincères condoléances. 